\title{
Role of echocardiography in sepsis and septic shock
}

\author{
Saraschandra Vallabhajosyula ${ }^{1,2}$, Abdelrahman M. Ahmed ${ }^{2}$, Pranathi R. Sundaragiri ${ }^{3}$ \\ ${ }^{1}$ Department of Cardiovascular Medicine, ${ }^{2}$ Division of Pulmonary and Critical Care Medicine, Department of Medicine, ${ }^{3}$ Division of Hospital \\ Internal Medicine, Department of Medicine, Mayo Clinic, Rochester, MN, USA \\ Correspondence to: Saraschandra Vallabhajosyula, MD. Department of Cardiovascular Medicine, Mayo Clinic, 200 First Street SW, Rochester, MN \\ 55905, USA. Email: Vallabhajosyula.Saraschandra@mayo.edu. \\ Provenance and Peer Review: This article was commissioned by the Editorial Office, Annals of Translational Medicine. The article did not undergo \\ external peer review. \\ Comment on: Lan P, Wang TT, Li HY, et al. Utilization of echocardiography during septic shock was associated with a decreased 28-day mortality: a \\ propensity score-matched analysis of the MIMIC-III database. Ann Transl Med 2019;7:662.
}

Submitted Jan 02, 2020. Accepted for publication Jan 16, 2020.

doi: 10.21037/atm.2020.01.116

View this article at: http://dx.doi.org/10.21037/atm.2020.01.116

Sepsis continues to be leading cause of mortality and morbidity during and after an intensive care unit (ICU) and hospital stay (1-7). Sepsis is associated with multiorgan failure involving respiratory, renal, neurological and hepatic dysfunction and injury $(1,8,9)$. In recent times, there has been an increasing recognition of cardiovascular dysfunction in sepsis (1-15). Cardiac dysfunction in sepsis is driven primarily by release of cytokines, mitochondrial dysfunction, and tissue hypoxia that leads to cardiac myocyte injury and death (5). New-onset left ventricular and right ventricular dysfunction is seen in nearly $20-60 \%$ of all septic patients and is associated with worse outcomes $(4,5,8,12,14)$. Often times, sepsis may complicate an alternate shock state, such as cardiogenic, obstructive or vasoplegic shock leading to worsening hemodynamic and vasopressor requirements (10). Historically, these patients typically required monitoring with a pulmonary artery catheter to aid in better understanding of central and calculated peripheral hemodynamics (16). However, the decline in the use of pulmonary artery catheters, the limited applicability of minimally invasive hemodynamic monitoring tools and the inability of markers of perfusion (lactate, central venous oxygen saturation) to differentiate shock states has led to a paucity of tools for measuring hemodynamic dysfunction $(16,17)$. It is in this context, that echocardiography has found a niche in the non-cardiac ICUs. With the advent of bedside pointof-care ultrasonography (POCUS) in the ICU, there is greater recognition and understanding of cardiovascular dynamics in sepsis and septic cardiomyopathy (18). Bedside echocardiography in septic shock patients can provide valuable information on fluid responsiveness, septic cardiomyopathy and may aid in changes to management strategies (19).

In this issue of the Annals of Translational Medicine, Lan and colleagues examined the impact of echocardiography on the outcomes of patients who presented with septic shock (20). This retrospective study conducted between 2001 to 2012, utilized data from the publicly available Medical Information Mart for Intensive Care-III database, which is a single-center database of 46,520 critically ill patients admitted to Beth Israel Deaconess Medical Center, Boston, Massachusetts (20). A total of 3,291 of septic shock patients were included in the study analysis and were then subsequently divided into those receiving echocardiography $(\mathrm{N}=1,291)$ within the first 24 hours of septic shock diagnosis versus otherwise $(\mathrm{N}=2,000)$. Using propensity-matched analysis for 1,289 pairs, the authors evaluated 28-day mortality as their primary outcome. Propensity matching was performed for baseline variables, comorbidity, Sequential Organ Failure Assessment (SOFA) scores, central venous pressure, and mechanical ventilation use. The cohort receiving echocardiography had lower 28 -day mortality (33.2\% vs. 37.7\%; hazard ratio 0.83 (95\% confidence interval, $0.73-0.95) ; \mathrm{P}=0.005$ ). These results were consistent in the sub-groups without repeat echocardiography and those receiving a pulmonary artery catheter. Compared to the cohort not receiving echocardiography, the cohort receiving echocardiography had higher total duration and rates of norepinephrine, greater use of pulmonary artery catheters and inotropes, 
but comparable rates of minimally-invasive hemodynamic monitoring and transfusion requirements.

The authors must be commended for their work addressing an important question. As they highlight, echocardiography is the ideal hemodynamic tool in critically ill patients with shock, septic or otherwise. However, there are certain limitations to this study that are worthy of further discussion. We have three major areas of discussion related to this study.

One, the last patient in this study was enrolled in 2012. This was before the publication of three large randomized trials in 2014 and 2015 that did not show any benefit of the early goal-directed therapy protocol over usual care (21). Furthermore, the new Sepsis-III criteria, introduced in 2016, identify a sicker spectrum of septic patients compared to the Angus criteria used in this study (22). Echocardiography in the past decade was largely under the purview of cardiologists and echocardiographers, who may not have been practicing intensivists (23). POCUS in the ICU was at its inception during this study period, and therefore lacked standardization and documentation (23). Therefore, given these considerations, it is important to recognize that the findings of this study may have limited applicability to the current era. Further validation of these study results in a more contemporary population are needed to see if the improvements in outcome are related to overall improvements in the processes of care as compared to specific information that may be obtained from the echocardiogram.

Two, the propensity-matching design used in this study needs further discussion. Though the authors admirably tried to control for confounding using this methodology, it is not a substitute for randomization. One can only match for what is available, and therefore, it is important to question what is missing. For example, prior data have shown that the cardiovascular SOFA has limited discrimination for hemodynamic assessment in septic patients already in shock (6). As noted in their secondary outcomes, the echocardiography cohort had higher duration and rates of norepinephrine usage suggestive of greater severity in the cardiovascular domain. Cumulative quantification of vasoactive medications is crucial in septic pateints with cardiomyopathy as they often receive additional inotropes as evidenced in this study (6). Lastly, the authors did not account for treatment limiting decisions, including the involvement of palliative and hospice care specialists, which have significant bearing on mortality as an outcome. Therefore, did we truly compare cohorts that were free of bias and completely similar? The critical care literature has many such examples of retrospective studies showing a 'positive signal' only to be disproven in subsequent randomized trials. Therefore, though propensity-matching is an excellent tool, its utility over and above traditional multivariable regression remains to be further studied.

Finally, the most important consideration, what did the echocardiogram show and what should we be looking at? Hemodynamic tools rarely show mortality benefit primarily due to their diagnostic nature. The information outputted through the echocardiogram needs careful review, contextualization to the patient's pathophysiology, and decisive action if truly indicated. There are multiple potential pitfalls in this cognitive chain of events. For example, though training in echocardiography has been standardized for many years for cardiologists, certification for critical care echocardiography started only in 2019 $(23,24)$. The translational gap between the cardiologist/ echocardiographer in the echocardiographic laboratory and the intensivist at the bedside needs to be further understood. Did the intensivist have access to the fluid responsiveness information, which is usually not provided on routine echocardiograms (17)? Did the cardiologist factor in the different mechanical ventilation and vasoactive medication settings that may determine cardiac function $(12,17)$ ? Was the intervention-fluid bolus, increase or change in vasoactive medications and mechanical ventilation settings, administration of diuretics-made in response to or independent of the echocardiographic data? Finally, what information on the echocardiogram truly mattered? Prior studies have shown limited applicability of traditional measures of systolic dysfunction $(13,14)$. Left ventricular diastolic dysfunction and right ventricular dysfunction appears to be related to clinical outcomes, however their measurement might be challenging in critically ill patients $(4,12,13,25)$. Though strain imaging has shown promise in these patients, it has limited applicability in the ICU and therefore needs further validation (14). There is a need to define septic cardiomyopathy with greater precision, a need to delineate relevant echocardiographic parameters in septic shock, and understand the echocardiographic correlates of outcome prediction in this population $(13,14)$.

In summary, the authors are to be commended for their efforts in highlighting the role of echocardiography not only on the management but also on the outcomes of septic shock patients. There is a crucial need for greater integration of echocardiography in the evaluation of 
critically ill patients and work similar to that by Lan et al. (20) are instrumental in providing this impetus.

\section{Acknowledgments}

Funding: Dr. Saraschandra Vallabhajosyula is supported by the Clinical and Translational Science Award (CTSA) Grant Number UL1 TR000135 from the National Center for Advancing Translational Sciences (NCATS), a component of the National Institutes of Health (NIH). Its contents are solely the responsibility of the authors and do not necessarily represent the official view of NIH.

\section{Footnote}

Conflicts of Interest: SV serves as an unpaid editorial board member of Annals of Translational Medicine from Mar 2020 to Feb 2022. The other authors have no conflicts of interest to declare.

Ethical Statement: The authors are accountable for all aspects of the work in ensuring that questions related to the accuracy or integrity of any part of the work are appropriately investigated and resolved.

Open Access Statement: This is an Open Access article distributed in accordance with the Creative Commons Attribution-NonCommercial-NoDerivs 4.0 International License (CC BY-NC-ND 4.0), which permits the noncommercial replication and distribution of the article with the strict proviso that no changes or edits are made and the original work is properly cited (including links to both the formal publication through the relevant DOI and the license). See: https://creativecommons.org/licenses/by-nc-nd/4.0/.

\section{References}

1. Kotecha A, Vallabhajosyula S, Coville HH, et al. Cardiorenal syndrome in sepsis: A narrative review. J Crit Care 2018;43:122-7.

2. Kotecha AA, Vallabhajosyula S, Apala DR, et al. Clinical Outcomes of Weight-Based Norepinephrine Dosing in Underweight and Morbidly Obese Patients: A PropensityMatched Analysis. J Intensive Care Med 2018. [Epub ahead of print].

3. Pandompatam G, Kashani K, Vallabhajosyula S. The role of natriuretic peptides in the management, outcomes and prognosis of sepsis and septic shock. Rev Bras Ter
Intensiva 2019;31:368-78.

4. Vallabhajosyula S, Geske JB, Kumar M, et al. Dopplerdefined pulmonary hypertension in sepsis and septic shock. J Crit Care 2019;50:201-6.

5. Vallabhajosyula S, Jentzer JC, Geske JB, et al. NewOnset Heart Failure and Mortality in Hospital Survivors of Sepsis-Related Left Ventricular Dysfunction. Shock 2018;49:144-9.

6. Vallabhajosyula S, Jentzer JC, Kotecha AA, et al. Development and performance of a novel vasopressordriven mortality prediction model in septic shock. Ann Intensive Care 2018;8:112.

7. Vallabhajosyula S, Sakhuja A, Geske JB, et al. Role of Admission Troponin-T and Serial Troponin-T Testing in Predicting Outcomes in Severe Sepsis and Septic Shock. J Am Heart Assoc 2017. doi: 10.1161/JAHA.117.005930.

8. Vallabhajosyula S, Gillespie SM, Barbara DW, et al. Impact of New-Onset Left Ventricular Dysfunction on Outcomes in Mechanically Ventilated Patients With Severe Sepsis and Septic Shock. J Intensive Care Med 2018;33:680-6.

9. Vallabhajosyula S, Trivedi V, Gajic O. Ventilation in acute respiratory distress syndrome: importance of low-tidal volume. Ann Transl Med 2016;4:496.

10. Jentzer JC, Vallabhajosyula S, Khanna AK, et al. Management of Refractory Vasodilatory Shock. Chest 2018;154:416-26.

11. Vallabhajosyula S, Deshmukh AJ, Kashani K, et al. TakoTsubo Cardiomyopathy in Severe Sepsis: Nationwide Trends, Predictors, and Outcomes. J Am Heart Assoc 2018; 7:e09160.

12. Vallabhajosyula S, Kumar M, Pandompatam G, et al. Prognostic impact of isolated right ventricular dysfunction in sepsis and septic shock: an 8-year historical cohort study. Ann Intensive Care 2017;7:94.

13. Vallabhajosyula S, Pruthi S, Shah S, et al. Basic and advanced echocardiographic evaluation of myocardial dysfunction in sepsis and septic shock. Anaesth Intensive Care 2018;46:13-24.

14. Vallabhajosyula S, Rayes HA, Sakhuja A, et al. Global Longitudinal Strain Using Speckle-Tracking Echocardiography as a Mortality Predictor in Sepsis: A Systematic Review. J Intensive Care Med 2019;34:87-93.

15. Vallabhajosyula S, Sakhuja A, Geske JB, et al. Clinical profile and outcomes of acute cardiorenal syndrome type- 5 in sepsis: An eight-year cohort study. PLoS One 2018;13:e0190965.

16. Marik PE. Obituary: pulmonary artery catheter 1970 to 2013. Ann Intensive Care 2013;3:38. 
17. Cecconi M, De Backer D, Antonelli M, et al. Consensus on circulatory shock and hemodynamic monitoring. Task force of the European Society of Intensive Care Medicine. Intensive Care Med 2014;40:1795-815.

18. Feng M, McSparron JI, Kien DT, et al. Transthoracic echocardiography and mortality in sepsis: analysis of the MIMIC-III database. Intensive Care Medicine 2018;44:884-92.

19. Orme RM, Oram MP, McKinstry CE. Impact of echocardiography on patient management in the intensive care unit: an audit of district general hospital practice. Br J Anaesth 2009;102:340-4.

20. Lan P, Wang TT, Li HY, et al. Utilization of echocardiography during septic shock was associated with a decreased 28-day mortality: a propensity score-matched analysis of the MIMIC-III database. Ann Transl Med 2019;7:662.

Cite this article as: Vallabhajosyula S, Ahmed AM, Sundaragiri PR. Role of echocardiography in sepsis and septic shock. Ann Transl Med 2020;8(5):150. doi: 10.21037/atm.2020.01.116
21. Rowan KM, Angus DC, Bailey M, et al. Early, GoalDirected Therapy for Septic Shock - A Patient-Level Meta-Analysis. N Engl J Med 2017;376:2223-34.

22. Kashyap R, Singh TD, Rayes H, et al. Association of septic shock definitions and standardized mortality ratio in a contemporary cohort of critically ill patients. J Crit Care 2019;50:269-74.

23. Brown SM, Sekiguchi H, Pinsky MR. A New Era in Critical Care Ultrasound: Professionalization. Ann Am Thorac Soc 2017;14:1747-9.

24. Ryan T, Berlacher K, Lindner JR, et al. COCATS 4 Task Force 5: Training in Echocardiography: Endorsed by the American Society of Echocardiography. J Am Soc Echocardiogr 2015;28:615-27.

25. Lanspa MJ, Gutsche AR, Wilson EL, et al. Application of a simplified definition of diastolic function in severe sepsis and septic shock. Crit Care 2016;20:243. 